\title{
EX-IN-Ausbildungen: Experienced Involvement - Pro \& Kontra
}

\section{Training Experienced Involvement - Pro \& Contra}

Pro

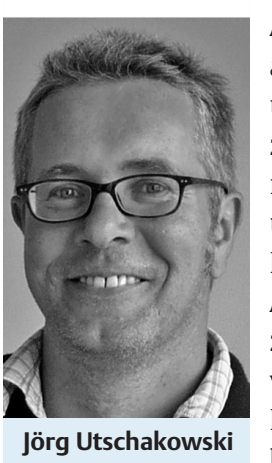

psychiatrischen Diensten veranstaltet oder unterstützt.

In der Schweiz wird das EX-IN-Curriculum sogar als DAS (Diploma of advanced studies) durchgeführt. Auf die 20 Studienplätze in diesem Jahr gibt es 70 Bewerbungen. Das Besondere an dem Studiengang ist nicht nur, dass EX-IN akademische Weihen erlangt hat, sondern dass mit den Abschlussarbeiten (zu Themen wie Recoverygruppen im psychiatrischen Krankenhaus, Umgang mit der Doppelrolle als Expertin durch Erfahrung und psychiatrischer Pflegefachkraft oder trialogische Interventionen in akuten Krisen) die Teilnehmer auch einen wichtigen Beitrag zur Fundierung und Verbreitung des Erfahrungswissens leisten.

schon in dem vorangegangenen europäischen Pilotprojekt deutlich, das von Beginn an von vielen kontroversen Diskussionen begleitet wurde. Zwei Jahre Zusammenarbeit von psychiatrieerfahrenen Menschen und psychiatrischen Fachkräften, von Vertretern von Forschungs- und Ausbildungseinrichtungen, Selbsthilfeorganisationen und psychiatrischen Diensten, die aus verschiedenen Ecken Europas kommen und das Ziel haben, am Ende eine gemeinsame Ausbildung zu beschreiben, war schon eine große Herausforderung. Alle waren von der Sinnhaftigkeit einer Qualifizierung von Experten durch Erfahrung überzeugt und auch von der Idee, dass dies nur gemeinsam gehen kann - nur über das Wie haben wir lange gerungen.

\section{Ausbildung}

Der Kernpunkt der EX-IN-Ausbildung ist der Austausch über Krisen- und Bewältigungserfahrungen, über Empowerment und Recovery, auf dessen Basis Erfahrungswissen entwickelt wird. Dieses Konzept, Erfahrungsexperten als Genesungsbegleiter und Dozenten zu qualifizieren, hat sich insbesondere im deutschsprachigen Raum erstaunlich schnell verbreitet.

Die Zeit für neue Formen der Beteiligung von psychiatrieerfahrenen Menschen scheint reif zu sein. In den 6 Jahren seit Erstellung des Curriculums wird der EX-INKurs in 14 von 16 Bundesländern an 20 Orten in Deutschland angeboten. Überall wird er von Netzwerken aus Selbsthilfeorganisationen Psychiatrieerfahrener und

\section{Nebenwirkungen}

Wenn wir uns hier mit dem Pro und Kontra von EX-IN beschäftigen, dann muss ich auch erwähnen, dass die Ausbildung nicht nur auf eine Tätigkeit als Genesungsbegleitung und Dozent vorbereitet, sondern auch den Teilnehmenden direkt zugute kommt. Die Motivationen, sich für einen der EX-IN-Kurse zu bewerben, sind sehr unterschiedlich, manche möchten einen Job, von dem sie leben können, andere wollen etwas zu ihrer Rente dazuverdienen, andere streben keine bezahlte Arbeit an, sondern wollen sich für ihre Selbsthilfetätigkeit weiter qualifizieren und manche möchten zunächst einfach etwas für sich tun.

Die Evaluation in der Pilotphase des Europaprojektes, die noch nicht veröffentlichte Auswertung des EX-IN-Studienganges durch die Universitätsklinik in Bern und die Aussagen der EX-IN-Trainer und Teilnehmer zeigen, dass für fast alle Kursteilnehmer die Ausbildung zu einem besseren Selbstwertgefühl, zu mehr Selbstbewusstsein und einer besseren Integration der Krisenerfahrung in das Selbstbild führt [1]. Allein dass Menschen wieder Mut finden, an sich zu glauben und ihr Leben in die Hand zu nehmen, spricht schon dafür, dass EX-IN ein Schritt in die richtige Richtung ist.

\section{Arbeit}

Bei der Konzipierung der EX-IN-Ausbilgrund, eine Qualifizierung für eine entdung stand von Anfang an im Vorder- lohnte Tätigkeit zu entwickeln. Die Idee, psychiatrieerfahrene Menschen als Genesungsbegleiter und Peer-Berater einzusetzen, war vor 6 Jahren in Deutschland noch relativ neu. Die ersten Stellen entstanden zunächst im betreuten Wohnen, in Wohnheimen und in Tagesstätten. Ein weiteres wichtiges Betätigungsfeld ist mittlerweile die integrierte Versorgung mit diversen ambulanten Angeboten, Home treatment und Krisenhäusern. Das Netzwerk psychische Gesundheit in Berlin beispielsweise beschäftigt in seinen 5 multiprofessionellen Teams 25\% EX-IN-Absolventen [2-4]. Nun ziehen erfreulicherweise auch die Kliniken nach. In Hamburg sind, unterstützt durch die Fördergelder als „Gesundheitsregion der Zukunft", in allen 8 Kliniken 25 EX-IN-Absolventen auf 400Euro-Basis beschäftigt [5]. Im Klinikum Reinkenheide/Bremerhaven arbeiten derzeit 4 Genesungsbegleiter mit je 30 Stunden wöchentlicher Arbeitszeit. Ziel ist dort, dass bis Ende 20124 weitere EX-INAbsolventen eingestellt werden, sodass alle 4 Stationen mit je 2 Genesungsbegleitern ausgestattet sind. Viele weitere Kliniken im gesamten Bundesgebiet haben ebenfalls bereits EX-IN-Absolventen eingestellt oder arbeiten daran. Die zunehmende Anzahl von Arbeitsplätzen wirkt sich auch positiv auf die Förderung der Ausbildung durch Job-Center und über das persönliche Budget aus.

\section{Was bringt der Einsatz von Genesungsbegleitern?}

Aus dem englischsprachigen Raum, wo der Einsatz von Erfahrungsexperten schon lange zur psychiatrischen Praxis gehört, wissen wir, dass sich die Qualität der psychiatrischen Angebote durch den Einsatz von Genesungsbegleitern verbessert. Insbesondere in den ersten 4 Wochen des Kontaktes zeigt sich, dass die Klienten schneller Vertrauen fassen und sich besser unterstützt fühlen. Auch die klinischen Ergebnisse sind oft besser als in den Vergleichsgruppen, die ohne Erfahrungsexperten arbeiten [6-8]. Die Genesungsbegleiter und Betroffenen können über Erlebnisse statt über Symptome reden. Es ist eher möglich, eine gemeinsame Sprache zu finden und eine von Akzeptanz, Verständnis und Empathie getragene Beziehung einzugehen. Die Experten durch 
Erfahrung können authentisch das Gefühl von Hoffnung und den Glauben an Weiterentwicklung vermitteln.

In Deutschland werden ähnliche Erfahrungen gemacht. Die Pflegedienstleitung aus dem Klinikum Reinkenheide berichtet: „Auch die Stationsleitungen bestätigen: Es gibt weniger Gewalt auf den Stationen. Stattdessen wird viel mehr miteinander gesprochen. Manchmal werden die Kollegen auch durch die Genesungsbegleiter gecoacht“ [9]. Leider gibt es bisher kaum Studien über die Auswirkungen der Arbeit der Erfahrungsexperten in Deutschland, aber durch die Begleitforschung im Rahmen des Hamburger Netzwerks psychische Gesundheit und durch den jüngst ins Leben gerufenen Forschungsverbund DACH (mit Universitäten aus Deutschland, Österreich und der Schweiz) werden bald gesicherte Erkenntnisse über die Auswirkungen der Kurse für die Teilnehmenden, die Arbeitsmarktchancen für die Absolventen und über die Auswirkungen des Einsatzes von Genesungsbegleitern für die Klienten vorliegen.

Deutlich ist jedoch schon jetzt, dass die Genesungsbegleiter wichtige Impulse für eine dialog- und genesungsorientierte psychiatrische Kultur bieten.

\section{Was bleibt zu tun?}

Natürlich sind wir noch nicht am Ende angekommen. Es bleiben noch viele Fragen zur Beschäftigung und Entlohnung offen. Wie können wir verbindliche Aufgabenbeschreibungen und tarifliche Einordnungen definieren, die verhindern, dass EXIN-Absolventen als billige Hilfskräfte eingesetzt werden? Wie können wir die
Standards der EX-IN-Ausbildung sichern und eine anerkannte Zertifizierung erreichen? $\mathrm{Zu}$ diesen und weiteren Anliegen ist jetzt der Verein EX-IN Deutschland gegründet worden. Zu seinen Aufgaben gehört auch, psychiatrische Dienste auf den Einsatz von Erfahrungsexperten vorzubereiten. Gemeinsam mit Kollegen aus Portugal, den Niederlanden, Belgien und Bulgarien haben wir dazu ein Handbuch entwickelt, das derzeit noch durch die Schweizer Erfahrungen ergänzt und im Sommer veröffentlicht wird.

Es gibt noch viel zu tun und jetzt scheint der richtige Zeitpunkt für weitere Schritte gekommen. Hätten wir gleich zu Beginn versucht, alle Fragen zu lösen, hätten wir meiner Meinung nach das Vorhaben überfrachtet, zu viel auf einmal gewollt, zu wenig Lobby gehabt. Jetzt ist EX-IN bundesweit bekannt, es gibt viele Erfahrungen mit der Ausbildung und der konkreten Arbeit von Genesungsbegleitern und es gibt viele engagierte Pioniere, die auf verschiedenen Ebenen sehr gute Modelle entwickelt haben. Für mich ist EX-IN eine Erfolgsgeschichte, die dazu beiträgt, die psychiatrische Versorgung zu verbessern und psychiatrieerfahrenen Menschen neue (berufliche) Perspektiven zu vermitteln.

\section{Literatur}

1 Hegedüs A, Steinauer R. Evaluation des Weiterbildungsstudiengangs DAS Experienced involvement. Erster internationaler Psychiatriekongress zu seelischer Gesundheit und Recovery 29. und 30. März 2012 in Bern. EX-IN Projektbericht 2007 (unveröffentlicht)

2 TAZ. Behandlung von Seelenkrankheiten. Den Stimmen zuhören. Im Konzept des „Hometreatment“ helfen ehemalige Psy- chiatriepatienten anderen Menschen mit seelischen Problemen in deren Krisen. vom 17.06.2010

3 Dribbusch B, Kleinschmidt M. „Aktuelle Ergebnisse aus der Umsetzung der IV-Verträge“. Fachtagung zum Stand der Integrierten Versorgung im NWpG 25.3.11, Berlin, Pinellodrom

4 Utschakowski U, Sielaff G, Bock T. Vom Erfahrenen zum Experten: Wie Peers die Psychiatrie verändern. Bonn: Psychiatrie Verlag; 2009

5 Eppendorfer. 4/2012: „Hamburg ist Weltspitze“: Startschuss für flächendeckende Peer-Beratung in den Psychiatrien.

6 Davidson L, Chinman M, Sells D et al. „Peer support among adults with serious mental illness: a report from the field“". Schizophrenia Bulletin 2006; 32: 443-450

7 Felton CJ, Statsny P, Shern DL et al. Consumers as Peer specialists on Intensive Case Management Teams: Impact on Client Outcomes. Psychiatric Sciences 1995; 46: 1037-1044

8 Greenfield TK, Stoneking BC et al. A Randomized Trial of a Mental Health Consumer-Managed Alternative to Civil Commitment for Acute Psychiatric Crisis. Community Psychol 2008; 42: 135 - 144

9 Interview des Autors mit Frau Lacroix, der Pflegedienstleitung in der Klinik für Psychiatrie und Psychotherapie am Klinikum Reinkenheide/Bremerhaven, am 20.04.12

Korrespondenzadresse

\section{Jörg Utschakowski}

Initiative zur sozialen Rehabilitation e. V. Waller Heerstraße 193

28219 Bremen

utschakowski@fokus-fortbildung.de

\section{Bibliografie}

DOI http://dx.doi.org/

10.1055/s-0032-1305015

Psychiat Prax 2012; 39: 202-203

(c) Georg Thieme Verlag KG

Stuttgart · New York

ISSN 0303-4259 